\title{
Spurious Harmonic Response of Multipulse Quantum Sensing Sequences
}

\author{
M. Loretz, ${ }^{1}$ J. M. Boss, ${ }^{1}$ T. Rosskopf, ${ }^{1}$ H. J. Mamin, ${ }^{2}$ D. Rugar, ${ }^{2}$ and C. L. Degen ${ }^{1, *}$ \\ ${ }^{1}$ Department of Physics, ETH Zurich, Otto Stern Weg 1, 8093 Zurich, Switzerland \\ ${ }^{2}$ IBM Research Division, Almaden Research Center, 650 Harry Road, San Jose, California 95120, USA
}

(Received 23 December 2014; published 22 April 2015)

\begin{abstract}
Multipulse sequences based on Carr-Purcell decoupling are frequently used for narrow-band signal detection in single-spin magnetometry. We have analyzed the behavior of multipulse sensing sequences under real-world conditions, including finite pulse durations and the presence of detunings. We find that these nonidealities introduce harmonics to the filter function, allowing additional frequencies to pass the filter. In particular, we find that the $X Y$ family of sequences can generate signals at the $2 f_{\mathrm{ac}}, 4 f_{\mathrm{ac}}$, and $8 f_{\mathrm{ac}}$ harmonics and their odd subharmonics, where $f_{\text {ac }}$ is the ac signal frequency. Consideration of the harmonic response is especially important for diamond-based nuclear-spin sensing where the nuclear magnetic resonance frequency is used to identify the nuclear-spin species, as it leads to ambiguities when several isotopes are present.
\end{abstract}

DOI: 10.1103/PhysRevX.5.021009

Subject Areas: Nanophysics, Physical Chemistry, Quantum Physics

\section{INTRODUCTION}

Multipulse decoupling sequences, initially developed in the field of nuclear magnetic resonance (NMR) spectroscopy [1,2], have enjoyed a renaissance for the control of individual quantum systems [3-6]. The concept relies on periodic reversals of the coherent evolution of the system, where the effect of the environment is canceled over the complete sequence. Multipulse decoupling can be regarded as an efficient high-pass filter that averages out lowfrequency noise.

It has recently been recognized that decoupling sequences with equal pulse spacing offer an opportunity for sensitive ac signal detection $[7,8]$. By tuning the interpulse delay $\tau$, the sequence can be made commensurate with a signal's periodicity leading to recoupling - that is, the decoupling fails for a specific set of signal frequencies that are an odd multiple of $1 /(2 \tau)$ [see Fig. 1(a)]. The quantum system then effectively acts as a narrow-band lock-in amplifier [9] with demodulation frequency $f=k /(2 \tau)$ and approximate bandwidth $f /(N / 2)$, where $N$ is the total number of pulses in the sequence and $k=1,3,5, \ldots$ is the resonance order. A precise transfer function of decoupling filters has been given in several recent papers $[7,9,10]$.

Multipulse sensing can greatly improve detection sensitivity, as it selectively measures the influence of a desired ac signal while suppressing unwanted noise. Moreover, the

\footnotetext{
*degenc@ethz.ch
}

Published by the American Physical Society under the terms of the Creative Commons Attribution 3.0 License. Further distribution of this work must maintain attribution to the author(s) and the published article's title, journal citation, and DOI. technique presents an opportunity to perform spectroscopy over a wide frequency range [10-14]. A particularly important application has been the detection of nanoscale NMR signals using single spins in diamond where the nuclear Larmor frequency is taken as a fingerprint for the detected spin species [15-25]. Although mostly developed in the context of single-spin magnetometry, multipulse sensing sequences have been applied to other quantum systems, including trapped ions [9] and superconducting qubits $[12,13]$.

In this paper, we consider a specific class of multipulse sensing sequences known as the $X Y$ family of sequences [26]. $X Y$-type sequences use the common template of equidistant $\pi$ pulses, but pulse phases are judiciously alternated so as to cancel out pulse imperfections, such as pulse amplitude variations or detuning. The $X Y$ family has become widely popular for measurements that require a large number of pulses $N$, and it has been the sequence of choice for most reported nitrogen-vacancy (NV)-based NMR experiments [19-25].

Here, we show that the phase alternation of $X Y$-type sequences causes additional frequencies to pass the multipulse filter. In particular, we show that an ac signal with frequency $f_{\text {ac }}$ will produce response at the second, fourth, and eighth harmonics (and their $k$ th subharmonics), depending on the sequence used. The harmonics are caused by a combination of time evolution during the finite duration of $\pi$ pulses and the "superperiods" introduced by phase cycling. Although the feature is generic to all experiments, it is most prominent for low pulse amplitudes and short interpulse delays, and for when a static detuning is present. The feature is significant because it leads to further ambiguities in signal analysis and complicates the interpretation of spectra. 


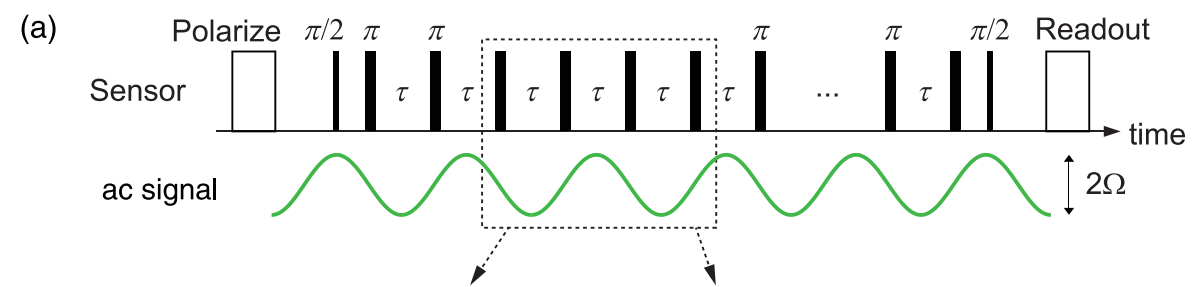

(b) $f=f_{\text {ac }}$

(c) $f=2 f_{\mathrm{ac}}$

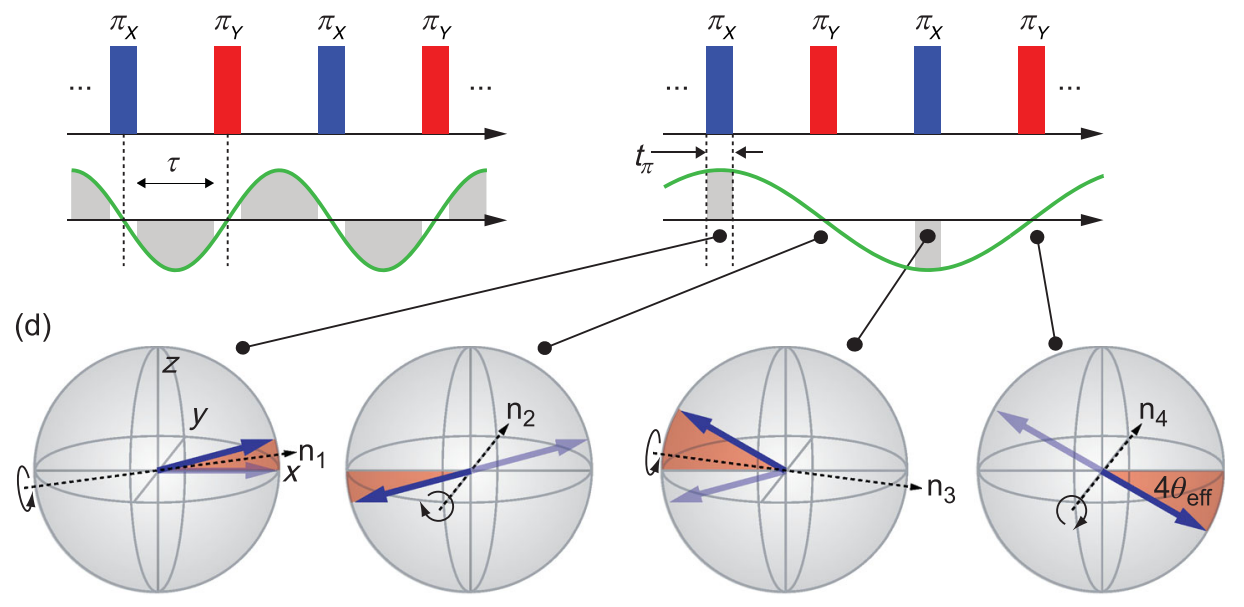

FIG. 1. (a) Generic scheme of multipulse quantum sensing based on Carr-Purcell decoupling. The detection frequency $f=1 /(2 \tau)$ is adjusted to match the ac signal frequency $f_{\mathrm{ac}}$. (b,c) Sensor phase accumulation for the $X Y 4$ sequence for the fundamental signal $f=f_{\text {ac }}$ and the second harmonic signal $f=2 f_{\text {ac }}$. Gray shaded areas show periods where phase accumulation occurs. (d) Bloch vector picture of the basic mechanism for phase accumulation at the second harmonic. $\mathbf{n}_{1 \ldots 4}$ are rotation axes of $\pi$ pulses, and $\theta_{\text {eff }}$ is the angle of the effective field, as explained in the text.

\section{THEORY}

In order to understand the generation of harmonics, we consider the simple case of an $X Y 4$ sequence exposed to an ac signal with frequency $f_{\text {ac }}$. The basic building block of the XY4 sequence consists of four $\pi$ pulses with alternating $X, Y$ phases [26] [see Figs. 1(b) and 1(c)]. In an ordinary sensing experiment, the interpulse spacing $\tau$ is matched to half the periodicity of the ac signal ( $f=f_{\text {ac }}$ ), leading to a constructive addition of phase during free evolution intervals. The phase accumulated per period $\tau$ is

$$
\phi_{\text {free }}=\frac{2 \Omega \tau}{\pi},
$$

and the phase accumulated during the entire sequence is $N \phi_{\text {free}}$, where $\Omega$ is the amplitude of the ac signal. $\Omega$ represents a coupling constant with units of angular frequency.

In any realistic experimental implementation, $\pi$ pulses have a finite duration, and additional time evolution of the quantum system will occur [27]. For the example of the $X Y 4$ sequence, we find that a feature of the additional time evolution is phase accumulation at the second harmonic where $f=2 f_{\text {ac }}$. This phase accumulation is different from $\phi_{\text {free }}$ in that it occurs during $\pi$ pulses and not during free evolution intervals.

The feature can be understood from a picture of Bloch vector rotations [Fig. 1(d)]. For simplicity, we assume that the ac field has maximum amplitude during $\pi_{X}$ pulses (and is zero during $\pi_{Y}$ pulses), and we neglect the free evolution during $\tau$. The action of the $X Y 4$ block can then be described by a set of four $\pi$ rotations $R_{\hat{\mathbf{n}}_{1 \ldots 4}}^{\pi}$ around the axes

$$
\begin{aligned}
& \hat{\mathbf{n}}_{\mathbf{1}}=\left(\cos \theta_{\text {eff }}, 0, \sin \theta_{\text {eff }}\right), \\
& \hat{\mathbf{n}}_{\mathbf{2}}=(0,1,0), \\
& \hat{\mathbf{n}}_{\mathbf{3}}=\left(\cos \theta_{\text {eff }}, 0,-\sin \theta_{\text {eff }}\right), \\
& \hat{\mathbf{n}}_{\mathbf{4}}=(0,1,0) .
\end{aligned}
$$

Axes $\hat{\mathbf{n}}_{\mathbf{1}}$ and $\hat{\mathbf{n}}_{\mathbf{3}}$ correspond to the $x$ axis tilted by the effective field angle $\theta_{\text {eff }}=\tan ^{-1}\left(\Omega / \omega_{1}\right) \approx \Omega / \omega_{1} \quad$ [2], where $\omega_{1}$ is the angular velocity (Rabi frequency) of $\pi$ rotations. The key feature here is that $\hat{\mathbf{n}}_{\mathbf{1}} \sharp \hat{\mathbf{n}}_{\mathbf{3}}$ because of the change in sign of the ac signal. Assuming the Bloch vector is initially aligned with the $x$ axis, the vector orientation after the four $\pi$ rotations is 
TABLE I. Anomalous phase $\phi_{\pi}$ at harmonics $f / f_{\text {ac }}$ for CPMG, $X Y 4, X Y 8$, and $X Y 16$ sequences (see Ref. [26] for sequence definition). Values were determined by a numerical simulation as described in Sec. IV. $\theta_{\text {eff }} \approx \Omega / \omega_{1}$ is the angle of the effective field. Entries marked by $*$ indicate that harmonics only appear in the presence of a static detuning.

\begin{tabular}{lcccccc}
\hline \hline Harmonic & 2 & 4 & 8 & $2 / 3$ & $4 / 3$ & $8 / 3$ \\
\hline CPMG & $\ldots$ & $\ldots$ & $\ldots$ & $\ldots$ & $\ldots$ & $\ldots$ \\
$X Y 4$ & $1.00 \theta_{\text {eff }}$ & $\ldots$ & $\ldots$ & $1.00 \theta_{\text {eff }}$ & $\ldots$ & $\ldots$ \\
$X Y 8$ & $0.71 \theta_{\text {eff }}$ & $0.27 \theta_{\text {eff }}$ & $\ldots$ & $0.71 \theta_{\text {eff }}$ & $0.65 \theta_{\text {eff }}$ & $\ldots$ \\
$X Y 16$ & $*$ & $*$ & $0.21 \theta_{\text {eff }}$ & $*$ & $*$ & $0.91 \theta_{\text {eff }}$ \\
\hline Harmonic & $2 / 5$ & $4 / 5$ & $8 / 5$ & $2 / 7$ & $4 / 7$ & $8 / 7$ \\
\hline CPMG & $\ldots$ & $\ldots$ & $\ldots$ & $\ldots$ & $\ldots$ & $\ldots$ \\
$X Y 4$ & $1.00 \theta_{\text {eff }}$ & $\ldots$ & $\ldots$ & $1.00 \theta_{\text {eff }}$ & $\ldots$ & $\ldots$ \\
$X Y 8$ & $0.71 \theta_{\text {eff }}$ & $0.65 \theta_{\text {eff }}$ & $\ldots$ & $0.71 \theta_{\text {eff }}$ & $0.27 \theta_{\text {eff }}$ & $\ldots$ \\
$X Y 16$ & $*$ & $*$ & $0.18 \theta_{\text {eff }}$ & $*$ & $*$ & $0.32 \theta_{\text {eff }}$ \\
\hline \hline
\end{tabular}

$$
R_{\hat{\mathbf{n}}_{4}}^{\pi} R_{\hat{\mathbf{n}}_{3}}^{\pi} R_{\hat{\mathbf{n}}_{2}}^{\pi} R_{\hat{\mathbf{n}}_{1}}^{\pi}(1,0,0)=\left(\cos 4 \theta_{\text {eff }}, 0,-\sin 4 \theta_{\text {eff }}\right) .
$$

This corresponds to a net rotation around the $y$ axis by an angle $4 \theta_{\text {eff }} \approx 4 \Omega / \omega_{1}$. The sensor therefore acquires an additional "anomalous" phase during the $X Y 4$ block, on average

$$
\phi_{\pi} \approx \theta_{\text {eff }} \approx \frac{\Omega}{\omega_{1}}=\frac{\Omega t_{\pi}}{\pi}
$$

per $\pi$ pulse, where $t_{\pi}$ is the duration of the square-shaped pulse. A similar calculation can be made for other harmonics and pulse sequences, and the resulting phases have been collected in Table I. These values are quantitative for the evolution during a single $X Y$ block in the limit of weak coupling, but they are only qualitative for longer sequences (due to backaction) or if a detuning is present.

The anomalous phase $\phi_{\pi}$ can be compared to the ordinary phase $\phi_{\text {free }}$, providing a "relative strength" $r=$ $\phi_{\pi} / \phi_{\text {free }}$ of harmonics compared to the fundamental signal. For the example of the second harmonic in $X Y 4$ detection,

$$
r=\frac{\phi_{\pi}}{\phi_{\text {free }}}=\frac{t_{\pi}}{2 \tau} .
$$

Other harmonics and sequences follow by using the appropriate multiplier from Table I. We find that the phase accumulated during $\pi$ rotations is equal to the phase accumulated during free evolution, scaled by $t_{\pi} / 2 \tau$. Since most often $t_{\pi} \ll \tau$, the harmonics will typically be much weaker than the fundamental signal. The harmonics may, however, become relevant if one intends to detect a weak ac signal in the presence of a strong, undesired signal. Note, finally, that higher order resonances $(k>1)$ are not attenuated (sometimes enhanced) for anomalous signals, unlike the ordinary signals where $\phi_{\text {free }} \propto 1 / k[15]$.

\section{SINGLE-SPIN MAGNETOMETRY}

The above considerations apply to several relevant situations in single-spin magnetometry of nanoscale NMR signals. Here, the precessing nuclear magnetization from single nuclei [15-17,25] or nuclear ensembles [19-24] provides the ac signal. In the following, we focus our attention on the specific case of an electron-nuclear two-spin system, where the electronic spin serves as the quantum sensor. The Hamiltonian of this system in a rotating frame of reference is

$$
\begin{aligned}
\hat{H}= & \underbrace{\Delta_{z} \hat{S}_{z}}_{\text {static }}+\underbrace{\omega_{1}\left\{x_{\bmod }(t) \hat{S}_{x}+y_{\bmod }(t) \hat{S}_{y}\right\}}_{\text {control }} \\
& +\underbrace{a_{\perp} \hat{S}_{z} \hat{I}_{x}+\omega_{0} \hat{I}_{z}}_{\text {ac signal }},
\end{aligned}
$$

where we have grouped terms into static, control, and ac contributions. $\Delta_{z}$ represents a static detuning of the electron spin $\hat{S}$ with respect to the rotating frame of reference, $x_{\text {mod }}(t)$ and $y_{\text {mod }}(t)$ represent the amplitude modulation of the multipulse decoupling sequence (with values between -1 and 1), $a_{\perp}$ represents the transverse coupling to the nuclear spin $\hat{I}$, and $\omega_{0}=\gamma_{n} B+a_{\|} \hat{S}_{z} \approx$ $\gamma_{n} B+a_{\|} / 2$ is the effective Larmor frequency of the nuclear spin composed of static bias field $B$ and parallel hyperfine field contribution $a_{\|}$(see supplemental material to Ref. [25] for a detailed discussion). $\gamma_{n}$ is the nuclear gyromagnetic ratio.

Returning to our generic expression for anomalous phase accumulation, Eq. (4), we can associate $\Omega \rightarrow$ $a_{\perp} / 2$ and $f_{\text {ac }} \rightarrow \omega_{0} /(2 \pi)$. For the case of a large nuclear-spin ensemble with rms nuclear field $B_{\mathrm{ac}}^{\text {rms }}$, one would associate $\Omega \rightarrow \gamma_{e} B_{\mathrm{ac}}^{\mathrm{rms}} / 2$, where $\gamma_{e}$ is the electron gyromagnetic ratio.

\section{SIMULATIONS}

We have performed a set of numerical simulations [25] to investigate the time evolution of the Hamiltonian, Eq. (6). Our quantity of interest was the probability that the spin state at the end of the sequence $|\alpha\rangle$ deviated from its original state $|0\rangle$, expressed by the transition probability $p=1-|\langle\alpha \mid 0\rangle|^{2}$.

Figure 2 presents simulated spectra for different multipulse sensing sequences. The top panel shows the filter response for ideal $\pi$ rotations of infinitely short duration. As expected, signal peaks are generated at frequencies $f=f_{\text {ac }} / k$, where $k=1,3,5, \ldots$. Lower panels, by contrast, show the filter response of $X Y$ sequences for real $\pi$ 


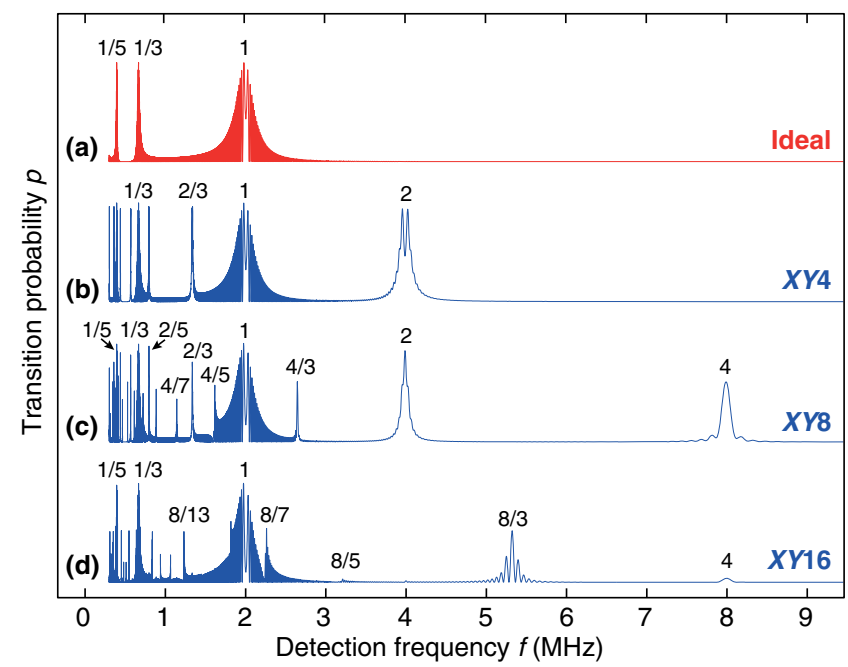

FIG. 2. Simulated spectra showing response as a function of detection frequency $f=1 /(2 \tau)$ for coupling to a single nuclear spin with $f_{\mathrm{ac}}=\omega_{0} /(2 \pi)=2 \mathrm{MHz}$ and $\Omega=a_{\perp} / 2=$ $2 \pi \times 200 \mathrm{kHz}$. (a) Ideal sensing sequence with infinitely short $\pi$ pulses. Only the expected peaks at $f_{\text {ac }} / k$ are observed. (b-d) Real $X Y 4, X Y 8$, and $X Y 16$ sequences [26] with finite, square-shaped $\pi$ pulses. Additional peaks at $2 f_{\mathrm{ac}} / k, 4 f_{\mathrm{ac}} / k$, and $8 f_{\mathrm{ac}} / k$ are observed, leading to a dense "forest" of peaks. The number of pulses is $N=480$, the Rabi frequency is $\omega_{1} / 2 \pi=20 \mathrm{MHz}$, and detuning (for c,d) is $\Delta_{z}=1 \mathrm{MHz}$.

rotations that have a finite duration. Many extra peaks appear corresponding to second, fourth, and eighth harmonics of $f_{\text {ac }} / k$. The spectra in Fig. 2 represent the response to a single ac signal (single nuclear spin) with frequency $f_{\mathrm{ac}}$. Obviously, if two or more signals were present, the analysis of the spectra would quickly become intractable.

Figure 3 investigates the influence of a static detuning $\Delta_{z}$. Several effects may be noticed. First, a static detuning exacerbates the harmonic peaks-a stronger anomalous response is generated for the same ac signal magnitude $\Omega$. Second, although not evident from this plot, peaks appear

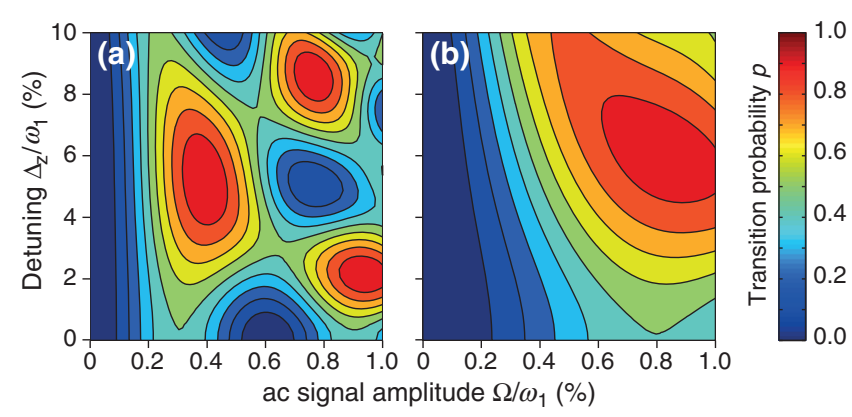

FIG. 3. Simulated transition probability for the (a) second and (b) fourth harmonics of the $X Y 8$ sequence. The horizontal axis is the ac signal amplitude, and the vertical axis is the detuning. Further simulation parameters are $N=1,024$ and $\omega_{1} / 2 \pi=$ $20 \mathrm{MHz}$. even at those harmonics where $\phi_{\pi}=0$ in Table I. Third, the time evolution of $p$ becomes markedly differentvalues oscillate in the range $p=0-0.5$ in the absence of a detuning, whereas they can oscillate in the range $p=0-1$ and become aperiodic in the presence of a detuning.

\section{EXPERIMENTS}

We have experimentally verified the existence of harmonics using the NV center in diamond as the single-spin sensor. The NV center is a prototype electron spin system $(S=1)$ that can be optically detected at room temperature [28] and that has served as a test bed for many recent multipulse sensing experiments. For our measurements, the $\mathrm{NV}$ center was polarized and read out using nonresonant green laser excitation and manipulated using microwave control pulses with adjustable phase and amplitude [20]. A static bias field was applied to lift the spin degeneracy, and all sensing experiments were carried out on the $m_{S}=0 \leftrightarrow m_{S}=-1$ subsystem.

In a first experiment, we have recorded the $X Y 8$ response of a NV center with two proximal ${ }^{13} \mathrm{C}$ nuclei $(I=1 / 2)$ in a bias field of $183 \mathrm{mT}$. The nuclear Larmor frequencies of the two ${ }^{13} \mathrm{C}$ nuclei in this field were $f_{\mathrm{ac}}=1.97 \mathrm{MHz}$ and $f_{\mathrm{ac}}^{\prime}=2.08 \mathrm{MHz}$. (The frequencies were slightly different because of a small parallel hyperfine contribution.) Figure 4 shows the spectral response over the frequency range of $f=1.5-9 \mathrm{MHz}$. We found that almost all harmonics in

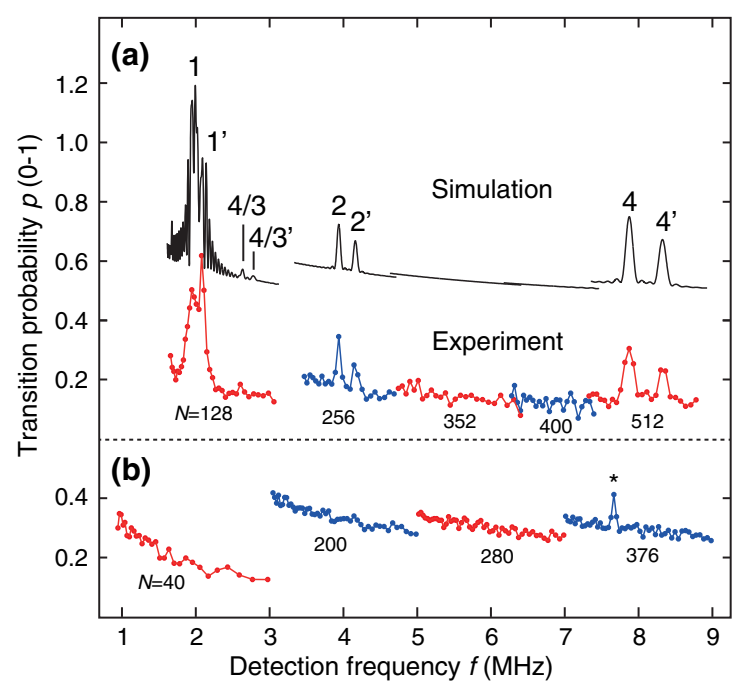

FIG. 4. (a) Spectrum recorded by a NV center with two weakly coupled ${ }^{13} \mathrm{C}$ nuclei, measured using an $X Y 8$ sequence. Multiple harmonics are clearly observed. Colors represent different data sets. Coupling constants for the two ${ }^{13} \mathrm{C}$ are $a_{\perp} / 2 \pi=220 \mathrm{kHz}$ and $a_{\perp}^{\prime} / 2 \pi=180 \mathrm{kHz}$. The number of pulses $N$ is given in the plot. The associated total evolution time is $T=N /(2 f)$. Further parameters are $\omega_{1} / 2 \pi=28 \mathrm{MHz}$ and $\Delta_{z}=1.5 \mathrm{MHz}$ (because of the ${ }^{15} \mathrm{~N}$ hyperfine splitting). (b) For comparison, the spectrum was recorded by a $\mathrm{NV}$ center with no ${ }^{13} \mathrm{C}$ nearby. The peak marked by $*$ is due to an ensemble of ${ }^{1} \mathrm{H}$ with $B_{\mathrm{ac}}^{\mathrm{rms}}=400 \mathrm{nT}$. 


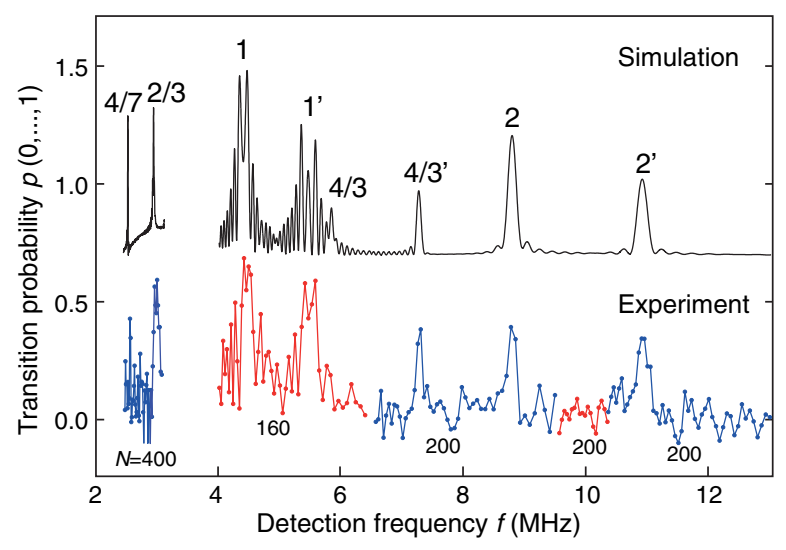

FIG. 5. Spectrum recorded by a NV center coupled to its own ${ }^{14} \mathrm{~N}$ nucleus, measured using an $X Y 8$ sequence. The common coupling constant is $a_{\perp} / 2 \pi=280 \mathrm{kHz}$. This coupling is due to a deliberate misalignment of the bias field of around $8^{\circ}$ with respect to the NV symmetry axis. Further experimental parameters are similar to Fig. 4.

that range could be resolved and matched with simulations using a single set of parameters.

Figure 5 shows a second example where the NV center's electron spin is coupled to its own ${ }^{14} \mathrm{~N}$ nucleus $(I=1)$. The ${ }^{14} \mathrm{~N}$ has two nuclear-spin transitions, resulting in two signals with frequencies $f_{\mathrm{ac}}=4.4 \mathrm{MHz}$ and $f_{\mathrm{ac}}^{\prime}=5.4 \mathrm{MHz}$. Again, most expected harmonics could be observed and matched with simulations.

\section{AMBIGUITIES BETWEEN NMR SIGNALS}

The feature of harmonics is of particular significance for recent nanoscale NMR experiments with near-surface NV centers. These experiments used spectral identification to discriminate different nuclear isotopes in samples by their NMR frequencies, most prominently ${ }^{1} \mathrm{H},{ }^{13} \mathrm{C},{ }^{19} \mathrm{~F},{ }^{29} \mathrm{Si}$, and ${ }^{31} \mathrm{P}$ [19-24].

We now show that in the presence of more than one nuclear species, harmonics can produce coincidental overlap between signals and lead to ambiguities in peak assignments. As a particular example, we consider the detection of a weak ${ }^{1} \mathrm{H}$ signal in the presence of ${ }^{13} \mathrm{C}$, which is a situation typical to $\mathrm{NV}$ centers in natural abundance diamond substrates (about $1 \%{ }^{13} \mathrm{C}$ content) [25]. Here, the signal overlap arises because the ${ }^{1} \mathrm{H}$ NMR frequency coincides with the $4 \times$ harmonic of ${ }^{13} \mathrm{C}$ to within $0.6 \%$. We find that single ${ }^{13} \mathrm{C}$ nuclei can generate signatures virtually identical to those of single ${ }^{1} \mathrm{H}$, including a Zeeman scaling with the magnetic field and a quantum-coherent oscillation of the signal.

We begin with the scaling of the peak frequency with bias field $B$, as shown in Fig. 6. The measured peak positions (points) can all be associated with the response from a single ${ }^{13} \mathrm{C}$ nucleus at either its fundamental Larmor frequency, or its second or fourth harmonic. The linear

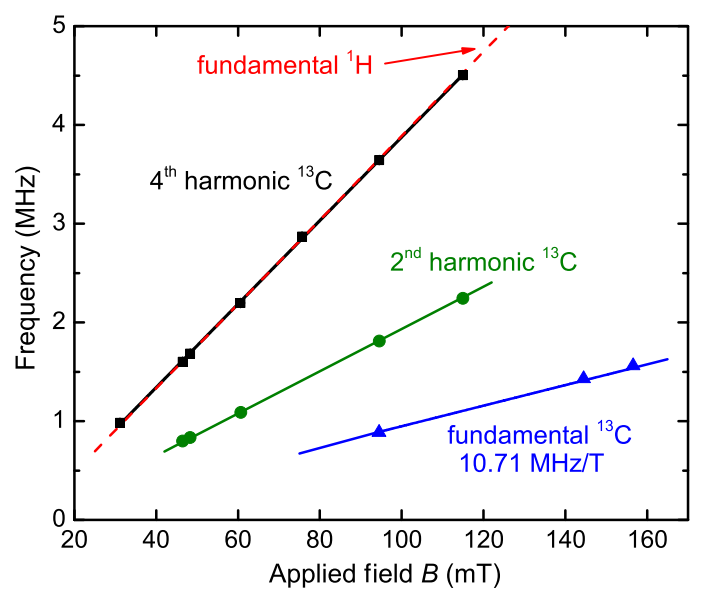

FIG. 6. Frequency of peak response for various magnetic bias fields $B$, measured using an $X Y 8$ sequence. Points are measured peak positions. Solid lines have slopes chosen to be 1,2 , and 4 times the gyromagnetic ratio of ${ }^{13} \mathrm{C}$. The red dashed line has a slope given by the gyromagnetic ratio of ${ }^{1} \mathrm{H}$ and is almost identical to the ${ }^{13} \mathrm{C}$ fourth harmonic line (black line).

slope of the fundamental frequency (blue line) is given by the gyromagnetic ratio of ${ }^{13} \mathrm{C}\left(\gamma_{n}=10.71 \mathrm{MHz} / \mathrm{T}\right)$ and is indicative of the nuclear species, while the slopes of the two harmonic frequencies are enhanced with apparent gyromagnetic ratios of $2 \gamma_{n}$ and $4 \gamma_{n}$. Since the value of $4 \gamma_{n}=42.82 \mathrm{MHz} / \mathrm{T}$ coincides with the gyromagnetic ratio of protons $\left(\gamma_{n}=42.57 \mathrm{MHz} / \mathrm{T}\right)$ within experimental uncertainty, the nuclear isotope cannot be uniquely identified.

We also found that the time evolutions of fundamental and harmonic signals produce indistinguishable signatures. Figure 7 shows the peak height of an apparent proton signal

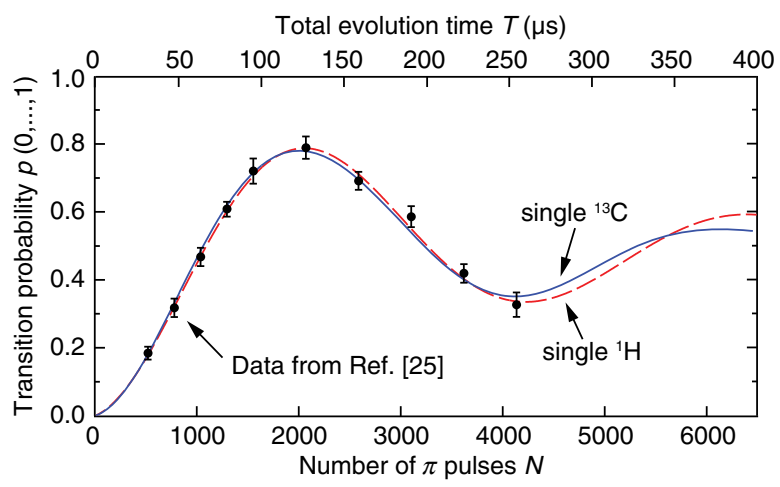

FIG. 7. Peak transition probability with increasing total evolution time $T=N \tau$ measured at the expected ${ }^{1} \mathrm{H}$ resonance. Black data points are taken from Ref. [25]. The red dashed curve shows simulation for a single ${ }^{1} \mathrm{H}$ spin with coupling constant $a_{\perp} / 2 \pi=14 \mathrm{kHz}$. The blue solid curve shows simulation for the fourth harmonic of a single ${ }^{13} \mathrm{C}$ spin with a coupling constant $a_{\perp} / 2 \pi=150 \mathrm{kHz}$. The pulse sequence is $X Y 8$. Further parameters are $B=182 \mathrm{mT}, \omega_{1} / 2 \pi=25 \mathrm{MHz}$, and $\tau=61.5 \mathrm{~ns}$. 
TABLE II. Examples of coincidental overlap between fundamental and harmonic frequencies for important NMR isotopes. Values are given for a 100-mT bias field. All of the harmonics listed are visible in Fig. 2.

\begin{tabular}{lccccc}
\hline \hline Isotope & Frequency & $\begin{array}{c}\text { Mimicking } \\
\text { isotope }\end{array}$ & Harmonic & $\begin{array}{c}\text { Harmonic } \\
\text { frequency }\end{array}$ & $\begin{array}{c}\text { Relative } \\
\text { frequency } \\
\text { difference }\end{array}$ \\
\hline${ }^{1} \mathrm{H}$ & $4.257 \mathrm{MHz}$ & ${ }^{13} \mathrm{C}$ & $4 \times$ & $4.282 \mathrm{MHz}$ & $0.6 \%$ \\
${ }^{29} \mathrm{Si}$ & $0.846 \mathrm{MHz}$ & ${ }^{1} \mathrm{H}$ & $1 / 5 \times{ }^{\mathrm{a}}$ & $0.851 \mathrm{MHz}$ & $0.7 \%$ \\
& & ${ }^{13} \mathrm{C}$ & $4 / 5 \times$ & $0.856 \mathrm{MHz}$ & $1.2 \%$ \\
${ }^{31} \mathrm{P}$ & $1.723 \mathrm{MHz}$ & ${ }^{1} \mathrm{H}$ & $2 / 5 \times$ & $1.703 \mathrm{MHz}$ & $1.2 \%$ \\
\hline \hline
\end{tabular}

${ }^{\mathrm{a}}$ Ordinary (not anomalous) harmonic.

as the number of pulses $N$ is increased. An oscillating signal is observed that can be reproduced by either of two simulations: The first assumes the presence of a single ${ }^{1} \mathrm{H}$, and the second assumes a single ${ }^{13} \mathrm{C}$. Again, no conclusion can be made on the identity of the nuclear species.

The above example illustrates that the assignment of a signal based on a single peak is, in general, insufficient. A more trustworthy identification could be obtained by measuring a wide spectral region containing several harmonics, as shown in Fig. 4. For the specific situation of ${ }^{13} \mathrm{C}$ and ${ }^{1} \mathrm{H}$, the second harmonic at twice the ${ }^{13} \mathrm{C}$ Larmor frequency (roughly half the ${ }^{1} \mathrm{H}$ frequency) could serve as a tell-tale signature of ${ }^{13} \mathrm{C}$. This peak is absent for ${ }^{1} \mathrm{H}$.

We note that the ambiguity between ${ }^{1} \mathrm{H}$ and ${ }^{13} \mathrm{C}$ is just one particular case. Given the large number of harmonics and nuclear isotopes, many other pairs of nuclear species with potential overlap can be expected. Table II collects a few additional cases of particular relevance to NMR where such coincidental signal overlap could occur. Consideration of harmonics is especially important in the presence of ${ }^{1} \mathrm{H}$ or for ${ }^{13} \mathrm{C}$-containing diamond because these species often produce a relatively strong signal.

\section{SUMMARY}

In summary, we have investigated the spectral filtering characteristics of an important class of multipulse quantum sensing sequences, the $X Y$ family of sequences. We found that the time evolution during finite $\pi$ pulses, which is present in any experimental implementation, causes phase buildup at higher harmonics of the signal frequency, leading to sets of additional peaks in the spectrum. We have further investigated this feature by simulations and experiments of single NV centers in diamond. The feature has particular significance for nanoscale NMR experiments that rely on spectral identification. Specifically, we found that signal detection at a single frequency is, in general, insufficient to uniquely assign a certain nuclear-spin species.

We finally mention several sensing schemes that do not suffer from the ambiguities inherent to multipulse sequences. Namely, these include rotating-frame spectroscopy [29-31] and free precession techniques [32-34]. Moreover, it may be possible to craft varieties of sequences that avoid harmonic resonances yet maintain superior static error compensation, such as sequences with composite $\pi$ pulses $[4,35]$, locally nonuniform pulse spacing [36], or aperiodic phase alternation. Although the alternative schemes have their own restrictions and may not always be available, they could offer independent verification of spectral features.

\section{ACKNOWLEDGMENTS}

This work was supported by Swiss NSF Project No. 200021_137520, the NCCR QSIT, the DIADEMS programme of the European Commission, and the DARPA QuASAR program.

[1] H. Y. Carr and E. M. Purcell, Effects of Diffusion on Free Precession in Nuclear Magnetic Resonance Experiments, Phys. Rev. 94, 630 (1954).

[2] C. P. Slichter, Principles of Magnetic Resonance (Springer, Heidelberg, 1996).

[3] J. Du, X. Rong, N. Zhao, Y. Wang, J. Yang, and R. B. Liu, Preserving Electron Spin Coherence in Solids by Optimal Dynamical Decoupling, Nature (London) 461, 1265 (2009).

[4] C. A. Ryan, J. S. Hodges, and D. G. Cory, Robust Decoupling Techniques to Extend Quantum Coherence in Diamond, Phys. Rev. Lett. 105, 200402 (2010).

[5] G. de Lange, Z. Wang, D. Riste, V. Dobrovitski, and R. Hanson, Universal Dynamical Decoupling of a Single Solid-State Spin from a Spin Bath, Science 330, 60 (2010).

[6] B. Naydenov, F. Dolde, L. T. Hall, C. Shin, H. Fedder, L. C. L. Hollenberg, F. Jelezko, and J. Wrachtrup, Dynamical Decoupling of a Single-Electron Spin at Room Temperature, Phys. Rev. B 83, 081201 (2011).

[7] G. De Lange, D. Riste, V. V. Dobrovitski, and R. Hanson, Single-Spin Magnetometry with Multipulse Sensing Sequences, Phys. Rev. Lett. 106, 080802 (2011).

[8] N. Zhao, J. L. Hu, S. W. Ho, J. T. K. Wan, and R. B. Liu, Atomic-Scale Magnetometry of Distant Nuclear Spin Clusters via Nitrogen-Vacancy Spin in Diamond, Nat. Nanotechnol. 6, 242 (2011).

[9] S. Kotler, N. Akerman, Y. Glickman, A. Keselman, and R. Ozeri, Single-Ion Quantum Lock-in Amplifier, Nature (London) 473, 61 (2011). 
[10] L. Cywinski, R. M. Lutchyn, C. P. Nave, and S. Das Sarma, How to Enhance Dephasing Time in Superconducting Qubits, Phys. Rev. B 77, 174509 (2008).

[11] G. A. Alvarez and D. Suter, Measuring the Spectrum of Colored Noise by Dynamical Decoupling, Phys. Rev. Lett. 107, 230501 (2011).

[12] J. Bylander, S. Gustavsson, F. Yan, F. Yoshihara, K. Harrabi, G. Fitch, D. G. Cory, Y. Nakamura, J.-S. Tsai, and W. D. Oliver, Noise Spectroscopy through Dynamical Decoupling with a Superconducting Flux Qubit, Nat. Phys. 7, 565 (2011).

[13] S. Gustavsson, F. Yan, J. Bylander, F. Yoshihara, Y. Nakamura, T. P. Orlando, and W.D. Oliver, Dynamical Decoupling and Dephasing in Interacting Two-Level Systems, Phys. Rev. Lett. 109, 010502 (2012).

[14] Y. Romach et al., Spectroscopy of Surface-Induced Noise Using Shallow Spins in Diamond, Phys. Rev. Lett. 114, 017601 (2015).

[15] T. H. Taminiau, J. J. T. Wagenaar, T. van der Sar, F. Jelezko, V. V. Dobrovitski, and R. Hanson, Detection and Control of Individual Nuclear Spins Using a Weakly Coupled Electron Spin, Phys. Rev. Lett. 109, 137602 (2012).

[16] N. Zhao et al., Sensing Single Remote Nuclear Spins, Nat. Nanotechnol. 7, 657 (2012).

[17] S. Kolkowitz, Q. P. Unterreithmeier, S. D. Bennett, and M. D. Lukin, Sensing Distant Nuclear Spins with a Single Electron Spin, Phys. Rev. Lett. 109, 137601 (2012).

[18] F. Shi, X. Kong, P. Wang, F. Kong, N. Zhao, R.-B. Liu, and J. Du, Sensing and Atomic-Scale Structure Analysis of Single Nuclear-Spin Clusters in Diamond, Nat. Phys. 10, 21 (2014).

[19] T. Staudacher, F. Shi, S. Pezzagna, J. Meijer, J. Du, C. A. Meriles, F. Reinhard, and J. Wrachtrup, Nuclear Magnetic Resonance Spectroscopy on a (5-Nanometer) ${ }^{3}$ Sample Volume, Science 339, 561 (2013).

[20] M. Loretz, S. Pezzagna, J. Meijer, and C. L. Degen, Nanoscale Nuclear Magnetic Resonance with a 1.9-nmDeep Nitrogen-Vacancy Sensor, Appl. Phys. Lett. 104, 033102 (2014).

[21] C. Muller et al., Nuclear Magnetic Resonance Spectroscopy with Single Spin Sensitivity, Nat. Commun. 5, 4703 (2014).

[22] S. J. Devience et al., Nanoscale NMR Spectroscopy and Imaging of Multiple Nuclear Species, Nat. Nanotechnol. 10, 129 (2015).

[23] D. Rugar, H. J. Mamin, M. H. Sherwood, M. Kim, C. T. Rettner, K. Ohno, and D. D. Awschalom, Proton Magnetic
Resonance Imaging Using a Nitrogen-Vacancy Spin Sensor, Nat. Nanotechnol. 10, 120 (2015).

[24] T. Haberle, D. Schmid-Lorch, F. Reinhard, and J. Wrachtrup, Nanoscale Nuclear Magnetic Imaging with Chemical Contrast, Nat. Nanotechnol. 10, 125 (2015).

[25] M. Loretz et al., Single-Proton Spin Detection by Diamond Magnetometry, Science, doi:10.1126/science.1259464 (2014).

[26] T. Gullion, D. B. Baker, and M. S. Conradi, New, Compensated Carr-Purcell Sequences, J. Magn. Reson. 89, 479 (1990).

[27] S. Pasini, P. Karbach, and G. S. Uhrig, High-Order Coherent Control Sequences of Finite-Width Pulses, Europhys. Lett. 96, 10003 (2011).

[28] F. Jelezko and J. Wrachtrup, Single Defect Centres in Diamond: A Review, Phys. Status Solidi (a) 203, 3207 (2006).

[29] F. Yan, S. Gustavsson, J. Bylander, X. Jin, F. Yoshihara, D. G. Cory, Y. Nakamura, T. P. Orlando, and W. D. Oliver, Rotating-Frame Relaxation as a Noise Spectrum Analyser of a Superconducting Qubit Undergoing Driven Evolution, Nat. Commun. 4, 2337 (2013).

[30] M. Loretz, T. Rosskopf, and C. L. Degen, Radio-Frequency Magnetometry Using a Single Electron Spin, Phys. Rev. Lett. 110, 017602 (2013).

[31] P. London et al., Detecting and Polarizing Nuclear Spins with Double Resonance on a Single Electron Spin, Phys. Rev. Lett. 111, 067601 (2013).

[32] H. J. Mamin, M. Kim, M. H. Sherwood, C. T. Rettner, K. Ohno, D. D. Awschalom, and D. Rugar, Nanoscale Nuclear Magnetic Resonance with a Nitrogen-Vacancy Spin Sensor, Science 339, 557 (2013).

[33] A. Laraoui, F. Dolde, C. Burk, F. Reinhard, J. Wrachtrup, and C. A. Meriles, High-Resolution Correlation Spectroscopy of C-13 Spins Near a Nitrogen-Vacancy Centre in Diamond, Nat. Commun. 4, 1651 (2013).

[34] T. H. Taminiau, J. Cramer, T. van der Sar, V. V. Dobrovitski, and R. Hanson, Universal Control and Error Correction in Multi-qubit Spin Registers in Diamond, Nat. Nanotechnol. 9, 171 (2014).

[35] A. M. Souza, G. A. Alvarez, and D. Suter, Robust Dynamical Decoupling for Quantum Computing and Quantum Memory, Phys. Rev. Lett. 106, 240501 (2011).

[36] N. Zhao, J. Wrachtrup, and R. B. Liu, Dynamical Decoupling Design for Identifying Weakly Coupled Nuclear Spins in a Bath, Phys. Rev. A 90, 032319 (2014). 